\title{
Renormalization Group Study of Sandpile on the Triangular Lattice.
}

\author{
Vl.V. Papoyan* \\ and \\ A.M. Povolotsky ${ }^{\dagger}$ \\ Bogoliubov Laboratory of Theoretical Physics, \\ JINR, 141980 Dubna, Russia.
}

\begin{abstract}
We apply the renormalization group approach to the sandpile on the triangular lattice. The only attractive fixed point is found. The obtained fixed point height probabilities are compared with numerical simulations. The value of critical exponent of avalanche size distribution is found to be $\tau=1.36$. The probabilities of the sand transition are compared with the branching probabilities of the spanning trees on the triangular lattice which are also evaluated.
\end{abstract}

*E-mail: vpap@theor.jinrc.dubna.su

†E-mail: povam@thsun1.jinr.dubna.su 


\section{Introduction.}

The main idea of the renormalization group ( $R G$ ) approach to critical fenomena is the calculation of parameters of a critical state by a successive transition from small-scale interactions to the large-scale ones. It is possible due to the vanishing of characteristic temporal and spatial scales at the critical point leading to temporal and spatial selfsimilarity. A wide range of the systems exhibiting these properties is unified by the concept of Self-Organized Criticality (SOC) introduced by Bak et al [1]. These systems are characterized by the evolution into the critical state free from fine tuning of any parameters. The sandpile model was proposed in the same work to illustrate the idea of the SOC. This cellular automaton was found to be rather a simple object of investigations, although rich in content. The sandpile model involves the set of sites on which the function of height is defined. Time after time being added to a random site, "grains of sand" cause the growth of its height. If the height reaches some critical value, the site topples reducing its height and increasing the heights of several neighboring sites. In turn, if the heights of the neighboring sites reach the critical value, they also topple and so on. The "sand" can leave the system on the boundary. After evolving for some time the system comes into the recurrent subset of all its configurations which is the critical state. Further the successive topplings between the stable states called avalanche return the system into the critical state each time it leaves it.

The most successful RG approach to the description of the sandpile on the square lattice has been proposed by Pietronero et al [2]. It is based on the real space RG scheme driven by steady state condition feedback. The real space renormalization is performed according to the standard block-transformation scheme. The feedback mechanism allows one to take into account the strong correlations appearing at the critical point. Practically, this approach identifies the dynamic and static parameters of the model. While the static configurations are described in terms of stable and critical cell distribution, the dynamic renormalized variables characterize the toppling rule for the critical cells. The conservation law valid in the critical state makes it possible to obtain the feedback relation linking critical values of the dynamic parameters and the static configuration weights. The obtained renormalization equation has a unique attractive nontrivial fixed point. By considering the avalanche as a relaxation process at the definite scale the exponent $\tau$ of the distribution of the avalanche size was expressed through the fixed point parameters.

The next step in this direction was made by Ivashkevich [4. While previous approach dealt only with the critical cell distribution, the cells with different heights were introduced into this model. Further, the sandpile evolution was identified with the branching process and the kinetic equations for the sand transfer were written as equations of chemical reactions of a special type. This finer consideration allows one to take into account more processes of toppling inside a cell. In addition, the generating function method proposed in the same work makes all calculations much simpler. Thus, the exponent $\tau$ was defined with higher accuracy, and different height site concentrations were calculated.

Due to the fixed point independence from the smallest scale dynamics, all the results are valid for the model with an arbitrary rule of toppling. Therefore, this is correct for 
the Abelian sandpile model (ASM), which was shown to be exactly soluble by Dhar [5]. In fact, the values of fixed point concentrations are, indeed, in good agreement with the exact values calculated by Priezzhev [6], and value $\tau$ obtained in [2] and [4] is very close to $\tau=1.25$ proposed by Priezzhev et al in [7].

The relationship between the configurations of the ASM and spanning trees was found in [8] for the first time. Later the one-to-one correspondence between the allowed ASM configurations and the set of spanning trees was shown in [9]. In the letter [4], one interesting fact has also been noted: the fixed point probabilities of the sand being transferred from the toppling cell to one,two, three or four neighboring cells were found to be almost equal to the "branching probabilities of spanning trees" which are the probabilities of a random site of the spanning tree having a coordination number 1,2,3 or 4, exactly calculated in [10]. It makes sense to verify the hypothesis about the equivalence of these probabilities and in this way to clarify a possible relation between the RG approach and the spanning tree statistics.

Excellent results of the RG approach on the square lattice call for obtaining the same results on other lattices. In our paper, we consider the $\mathrm{RG}$ approach for the triangular lattice. We compare the obtained equilibrium concentrations with the computer simulations and the exponent $\tau$ of the avalanche size distribution with its exact value. We also calculate the branching probabilities of the spanning trees on the triangular lattice and compare them with the fixed point probabilities of the sand transfer into neighboring sites. It is shown that the hypothesis about their coincidence at least does not have a universal character.

\section{Renormalization approach.}

Let us consider a sandpile on the triangular lattice. The height of each site can take an integer value $Z=1,2, \ldots$. If the height of any site exceeds the critical value $Z_{c}=6$, it topples. At this moment, the particles can be transferred to one or several neighboring sites, increasing their heights by one. This toppling rule is characterized by the vector $\mathbf{p}=\left(p_{1}, p_{2}, \ldots, p_{6}\right)$, where $p_{i}$ is the probability for the site to topple into $i$ neighboring cells.

Once we want to bring the consideration of this model from the smallest scale to a large one, we should define the successive scale increasing procedure. The step of this procedure consists in the transition from the sublattice $L_{b}$, which is constructed of the

cells of size $b$ on the initial lattice $L$, to the sublattice $L_{b \sqrt{3}}$ with the cells of size $b \sqrt{3}$. To realize it, three cells of the sublattice $L_{b}$ are unified into the block. This block is replaced by the cell of $L_{b \sqrt{3}}$, as is shown in Fig.1a. All bonds of $L_{b}$ outgoing from the given block and coming into another block are included into one renormalized bond of $L_{b \sqrt{3}}$, as is shown in Fig $1 \mathrm{~b}$.

Now, we must describe the coarse grained sandpile dynamics. In the following calculations we use the RG approach in the form proposed in [2] and [4]. Let us define the coarse grained variables for an arbitrary scale. They are introduced in such a way that 
one can treat the sandpile properties in any scale like ones of the original sandpile. Each cell of $L_{b}$ is characterized by two vectors. The first one is $\mathbf{n}^{(b)}=\left(n_{A}, n_{B}, n_{C}, n_{D}, n_{E}, n_{F}\right)$. This is the set of probabilities for the $L_{b}$ cell to behave, with respect to transformations initiated by the dropped into the cell particle, like a site on the initial lattice with height $1,2,3,4,5$ or 6 , respectively. In particular, $n_{F}$ is the probability that the cell is critical. The second vector, $\mathbf{p}^{(b)}$ is the analogue of the vector $\mathbf{p}$ in the initial sandpile.

Let us represent the coarse grained dynamics of the sandpile as the following branching process.

$$
\begin{aligned}
& \mathrm{A}+\varphi \rightarrow \mathrm{B}, \\
& \mathrm{B}+\varphi \rightarrow \mathrm{C}, \\
& \mathrm{C}+\varphi \rightarrow \mathrm{D}, \\
& \mathrm{D}+\varphi \rightarrow \mathrm{E}, \\
& \mathrm{E}+\varphi \rightarrow \mathrm{F}, \\
& \mathrm{F}+\varphi \rightarrow \begin{cases}p_{1}: & \mathrm{F}+\tilde{\varphi} \\
p_{2}: & \mathrm{E}+2 \tilde{\varphi} \\
p_{3}: & \mathrm{D}+3 \tilde{\varphi} \\
p_{4}: & \mathrm{C}+4 \tilde{\varphi} \\
p_{5}: & \mathrm{B}+5 \tilde{\varphi} \\
p_{6}: & \mathrm{A}+6 \tilde{\varphi} .\end{cases}
\end{aligned}
$$

Here the species $A, B, C, D, E, F$ are the cells which behave like the sites with heights $1,2,3,4,5,6$, and $\varphi, \tilde{\varphi}$ are the particles obtained by the cell and transferred into other cells, respectively. This branching process can be reinterpreted as irreversible chemical reactions for which we can write the kinetic equations of the transport of "sand" :

$$
\begin{aligned}
\dot{n}_{\mathrm{A}} & =n_{\varphi}\left(p_{6} n_{\mathrm{F}}-n_{\mathrm{A}}\right) \\
\dot{n}_{\mathrm{B}} & =n_{\varphi}\left(p_{5} n_{\mathrm{F}}+n_{\mathrm{A}}-n_{\mathrm{B}}\right) \\
\dot{n}_{\mathrm{C}} & =n_{\varphi}\left(p_{4} n_{\mathrm{F}}+n_{\mathrm{B}}-n_{\mathrm{C}}\right) \\
\dot{n}_{\mathrm{D}} & =n_{\varphi}\left(p_{3} n_{\mathrm{F}}+n_{\mathrm{C}}-n_{\mathrm{D}}\right) \\
\dot{n}_{\mathrm{E}} & =n_{\varphi}\left(p_{2} n_{\mathrm{F}}+n_{\mathrm{D}}-n_{\mathrm{E}}\right) \\
\dot{n}_{\mathrm{D}} & =n_{\varphi}\left(p_{1} n_{\mathrm{F}}+n_{\mathrm{E}}-n_{\mathrm{F}}\right) \\
\dot{n}_{\varphi} & =n_{\varphi}\left(\bar{p} n_{\mathrm{F}}-1\right)+\bar{p} \nu \nabla^{2}\left(n_{\varphi} n_{\mathrm{F}}\right)+\eta(\mathbf{r}, t) \\
\bar{p} & =6 p_{6}^{*}+5 p_{5}^{*}+4 p_{4}^{*}+3 p_{3}^{*}+2 p_{2}^{*}+p_{1}^{*}
\end{aligned}
$$

Here $n_{\varphi}$ denotes the concentrations of particles $\varphi, \eta(\mathbf{r}, t)$ is the noise term corresponding to the randomly dropping particles, $\nu$ is the diffusion coefficient, which is equal to $\frac{1}{6}$ for the triangle lattice, $\bar{p}$ is the average number of particles outgoing from the toppling cell.

Performing the renormalization procedure we express the parameters of $L_{b \sqrt{3}}$ through ones of $L_{b}$.

$$
\mathbf{n}^{(b \sqrt{3})}=\mathbf{n}\left(\mathbf{n}^{(b)}, \mathbf{p}^{(b)}\right), \quad \mathbf{p}^{(b \sqrt{3})}=\mathbf{p}\left(\mathbf{n}^{(b)}, \mathbf{p}^{(b)}\right)
$$


The critical values of $\mathbf{p}^{b}$ and $\mathbf{n}^{b}$ obtained by taking the limit $b \rightarrow \infty$ satisfy the renormalization equations

$$
\mathbf{n}^{*}=\mathbf{n}\left(\mathbf{n}^{*}, \mathbf{p}^{*}\right), \quad \mathbf{p}^{*}=\mathbf{p}\left(\mathbf{n}^{*}, \mathbf{p}^{*}\right)
$$

Due to nonlocal properties of the sandpile dynamics, writing down the first renormalization equation does not seem possible. To avoid it, we can exclude $\mathbf{n}^{*}$ from the second one. We use equations (2) and the requirement for the SOC state to be stationary. Taking

$$
\dot{\mathbf{n}}^{*}=0
$$

we can directly obtain

$$
\begin{aligned}
\mathrm{n}_{\mathrm{A}}^{*} & =\frac{p_{6}^{*}}{\bar{p}} \\
\mathrm{n}_{\mathrm{B}}^{*} & =\frac{p_{6}^{*}+p_{5}^{*}}{\bar{p}} \\
\mathrm{n}_{\mathrm{C}}^{*} & =\frac{p_{6}^{*}+p_{5}^{*}+p_{4}^{*}}{\bar{p}} \\
\mathrm{n}_{\mathrm{D}}^{*} & =\frac{p_{6}^{*}+p_{5}^{*}+p_{4}^{*}+p_{3}^{*}}{\bar{p}} \\
\mathrm{n}_{\mathrm{E}}^{*} & =\frac{p_{6}^{*}+p_{5}^{*}+p_{4}^{*}+p_{3}^{*}+p_{2}^{*}}{\bar{p}} \\
\mathrm{n}_{\mathrm{F}}^{*} & =\frac{p_{6}^{*}+p_{5}^{*}+p_{4}^{*}+p_{3}^{*}+p_{2}^{*}+p_{1}^{*}}{\bar{p}}=\frac{1}{\bar{p}} .
\end{aligned}
$$

Substituting this into the second equation (4) we obtain a closed renormalization equation for the vector $\mathbf{p}$. Search for the relationship between $\mathbf{p}^{(b)}$ and $\mathbf{p}^{(b \sqrt{3})}$ is reduced to counting the processes at the scale $b$ leading to the toppling of the cell at the scale $b \sqrt{3}$. To this end, we, following [4], introduce the generating function of the toppling of the cell at an arbitrary scale. If we denote six directions outgoing from the cell by $x_{1}, \ldots, x_{6}$, the generating function of toppling of the sublattice $L^{b}$ cell will be given by the formula

$$
\begin{gathered}
\sigma\left(x_{1}, x_{2}, x_{3}, x_{4}, x_{5}, x_{6}\right)=\frac{p_{1}^{(b)}}{6}\left(x_{1}+x_{2}+x_{3}+x_{4}+x_{5}+x_{6}\right)+ \\
\frac{p_{2}{ }^{(b)}}{15}\left(x_{1} x_{2}+x_{1} x_{3}+x_{1} x_{4}+\ldots+x_{5} x_{6}\right)+\frac{p_{3}{ }^{(b)}}{20} \sigma^{\prime \prime \prime}+ \\
\frac{p_{4}{ }^{(b)}}{15} \sigma^{\prime \prime \prime \prime}+\frac{p_{5}{ }^{(b)}}{6} \sigma^{\prime \prime \prime \prime \prime \prime}+p_{6}{ }^{(b)}\left(x_{1} x_{2} x_{3} x_{4} x_{5} x_{6}\right),
\end{gathered}
$$

where $\sigma$ with $i$ primes is the sum of all possible products $x_{k_{1}} x_{k_{2}} \ldots x_{k_{i}}$ of $i$ different terms $x_{k_{1}}, \ldots, x_{k_{i}}$. This function counts all processes leading to sand transition from the cell of $L_{b}$. The coefficient of each polynomial term is the probability for particles to go to the corresponding directions after the cell toppling. This function has the following properties:

a) If the arguments corresponding to any directions are replaced by zero, the function counts the toppling processes that don't send the particles to these directions. 
b) If they are replaced by unit, the function counts the toppling processes irrespective of outgoing to these directions.

c) If we want to describe the toppling of a couple of cells, where the particle sent by the first cell induces the toppling of the second one, we must replace the argument labeling the direction of outgoing of this particle by the $\sigma$-function of toppling of the second cell.This function contains also the processes in which the particle does not go in the given direction and the second cell does not topple. It is necessary to subtract them, if we want to take into account only processes spreading to both cells.

d) In addition, it is easy to see that the function is normalized so that $\sigma(1, \ldots, 1)=1$.

Combining $\sigma$-functions in different ways we can build the functions that count the specific topplings. Once we manage to link the generating functions at different scales, we express the probabilities $\mathbf{p}$ at a given scale through ones at a new scale. To this end, we write the generating function for the block consisting of three cells of $L_{b}$. This function must count the processes leading to the transition of particles from this block to some neighboring blocks. We can perform this using $\sigma$-function properties outlined above. In addition, according to [2] we must consider only processes which match the spanning condition, i.e. with the characteristic size equal to the size of a cell. In our case, these are relaxation processes in which more than one site of $L_{b}$ participates. All three types of blocks, whose topplings match the spanning condition and their relaxation schemes are shown in Fig 2. The rest of the schemes can be obtained from the shown ones by rotations.

For going over to the sublattice $L_{b \sqrt{3}}$, we replace the block considered by the cell of $L_{b \sqrt{3}}$, respectively renaming the outgoing directions, as is shown in Fig. 1b. To give the obtained function the meaning of the generating function, we must subtract all processes not going out of the block and normalize the result. Thus, we obtain the generating functions $\Sigma_{j}\left(y_{1}, y_{2}, y_{3}, y_{4}, y_{5}, y_{6}\right)(j=a, b, c)$ for the topplings of blocks of three kinds: $a, b$ and $c$. For example, the generating function for relaxation of the block of type $a$ is the following:

$$
\Sigma_{a}\left(y_{1}, y_{2}, y_{3}, y_{4}, y_{5}, y_{6}\right)=\frac{\tilde{\Sigma}_{a}\left(y_{1}, \ldots, y_{6}\right)-\tilde{\Sigma}_{a}(0, \ldots, 0)}{\tilde{\Sigma}_{a}(1, \ldots, 1)-\tilde{\Sigma}_{a}(0, \ldots, 0)}
$$

where

$$
\begin{aligned}
& \tilde{\Sigma}_{a}\left(y_{1}, y_{2}, y_{3}, y_{4}, y_{5}, y_{6}\right)= \\
& \left(\sigma\left(y_{1}, \sigma\left(y_{2}, y_{2}, y_{3}, 1,1, y_{1}\right), 1, y_{5}, y_{6}, y_{6}\right)-\sigma\left(y_{1}, 0,1, y_{5}, y_{6}, y_{6}\right)+\right. \\
& \left.\sigma\left(y_{2}, y_{2}, y_{3}, 1, \sigma\left(y_{1}, 1,1, y_{5}, y_{6}, y_{6}\right), y_{1}\right)-\sigma\left(y_{2}, y_{2}, y_{3}, 1,0, y_{1}\right)\right)+c . p .
\end{aligned}
$$

The arguments $y_{1} \ldots y_{6}$ denote the directions on the lattice at the new scale. The terms c.p. are obtained from the first term by the cyclic permutations of arguments corresponding to the rotation by the angles $\frac{2 \pi}{3}$ and $\frac{4 \pi}{3}$. The generating functions for the blocks of other types look analogously but they are too cumbersome to be given here. To write a general generating function, we accomplish summation over all types of blocks. Different types of blocks appear with the different probabilities which depend only on their heights. 
Therefore, we must multiply their generating functions by the corresponding weights. After summation the result must again be normalized

$$
\begin{gathered}
\Sigma\left(y_{1}, y_{2}, y_{3}, y_{4}, y_{5}, y_{6}\right)=\frac{\tilde{\Sigma}\left(y_{1}, \ldots, y_{6}\right)}{\tilde{\Sigma}(1, \ldots, 1)} \\
\tilde{\Sigma}=\mathrm{w}_{\mathrm{a}} \tilde{\Sigma}_{a}+\mathrm{w}_{\mathrm{b}} \tilde{\Sigma}_{b}+\mathrm{w}_{\mathrm{c}} \tilde{\Sigma}_{c} \\
\mathrm{w}_{a}=3 n_{\mathrm{F}}^{2}\left(n_{\mathrm{A}}+n_{\mathrm{B}}+n_{\mathrm{C}}+n_{\mathrm{D}}\right), \quad \mathrm{w}_{b}=3{n_{\mathrm{F}}}^{2} n_{\mathrm{E}}, \quad \mathrm{w}_{c}=n_{\mathrm{F}}{ }^{3} .
\end{gathered}
$$

Thus, we have the normalized generating function describing the toppling of a cell of the lattice $L_{b \sqrt{3}}$. According to the RG ideology, all particles outgoing from a cell in some direction become one renormalized particle. Therefore, the second powers of $y_{i}$ must be changed to the first powers. Now the coefficient of each term is again the probability of the respective process of toppling of the size $b \sqrt{3}$ cell. Taking the coefficients of sums analogous to $(1)$, we obtain new probabilities $\mathbf{p}^{(b \sqrt{3})}$ expressed through $\mathbf{p}^{(b)}$ and $\mathbf{n}^{(b)}$. Eventually, we have sought a system of renormalization equations. Solving this system, we obtain the fixed point values of $\mathbf{p}$ and $\mathbf{n}$. They are shown in tables 1 and 2 .

According to Pietronero et al, the critical exponent of the avalanche distribution $\tau$ may be expressed through the critical point parameters as follows. The probability of an avalanche of the linear size $r$ is $P(r) d r=r^{(1-2 \tau)} d r$. Thus, the probability for the avalanche to cover the cell of the size $b$ dying beyond it is given by the formula:

$$
K=\frac{\int_{b}^{b \sqrt{3}} P(r) d r}{\int_{b}^{\infty} P(r) d r}=1-(\sqrt{3})^{2(1-\tau)}
$$

On the other hand, $K$ can be expressed through the fixed point parameters

$$
K=p_{1}^{*}\left(1-n_{F}\right)+p_{2}^{*}\left(1-n_{F}^{*}\right)^{2}+\ldots+p_{6}^{*}\left(1-n_{F}^{*}\right)^{6}
$$

Using these expressions we obtain

$$
\tau=1-\frac{\ln (1-K)}{\ln 3}=1.366
$$

\section{Results and discussion.}

Obtaining the height probabilities in the sandpile on the triangular lattice by the $\mathrm{RG}$ approach seems to be efficient because of the absence of an exact result. While the RG approach is relatively simple, the exact calculations require applying a very complex technique even in the simplest case. In Table 1, we compare the fixed point height probabilities with the results of numerical simulation. One can notice a very similar behavior of the numerical and RG results. 


\begin{tabular}{lcccccc}
\hline \hline & $n_{1}^{*}$ & $n_{2}^{*}$ & $n_{3}^{*}$ & $n_{4}^{*}$ & $n_{5}^{*}$ & $n_{6}^{*}$ \\
\hline RG & 0.036 & 0.135 & 0.197 & 0.209 & 0.210 & 0.210 \\
Numerical & 0.054 & 0.092 & 0.139 & 0.188 & 0.240 & 0.281 \\
\hline \hline
\end{tabular}

TABLE 1. - Fixed point height probabilities in comparison with the numerical results. The numerical simulations were performed on the lattice $300 \times 300$ with statistics $10^{6}$ configurations.

Some distinction of their values is stipulated by initial roughness of the RG approach concerned with discrete increasing of the scale and averaged consideration of the coarse grained dynamics. However, the advantage of this RG scheme is a quite attractive character of the obtained fixed point.

The obtained value $\tau=1.366$ is close to the exact one 1.25 although the coincidence is rather worse than in the square lattice. This can be explained by a small size of a renormalized cell. The renormalization scheme thus constructed takes into account only a minimal number of toppling processes. Therefore, these calculations don't give high accuracy. However, this result allows us to speak about the stability for a given RG.

At the end, in Table 2, we compare the fixed point probabilities of the sand transfer $p_{k}$ with the branching probabilities of spanning trees calculated in Appendix.

\begin{tabular}{lcccccc}
\hline \hline & $p_{1}^{*}$ & $p_{2}^{*}$ & $p_{3}^{*}$ & $p_{4}^{*}$ & $p_{5}^{*}$ & $p_{6}^{*}$ \\
\hline RG & 0.0000179 & 0.00226 & 0.0558 & 0.296 & 0.471 & 0.174 \\
$\begin{array}{l}\text { Branching } \\
\text { probabilities }\end{array}$ & 0.322 & 0.417 & 0.207 & 0.0488 & 0.00553 & 0.000241 \\
\hline \hline
\end{tabular}

TABLE 2. - Fixed point probabilities $p_{k}$ of the sand being transported to $\mathrm{k}$ neighboring cells and branching probabilities of spanning trees on the triangular lattice.

The comparison of the probabilities of the sand transfer with the branching probabilities of spanning trees is very surprizing. As these quantities for the square lattice are very close, a similar behavior can be expected in our case as well. However, one can see quite an inverse order of the obtained values for the example considered.

Thus, we can conclude that the hypothesis about their coincidence is not confirmed in the case of triangular lattice. Further clarification of this question seems to be related to more complete investigations of a sandpile on a square lattice.

\section{Acknowledgments}

We are grateful to Prof. V.B. Priezzhev and E.V. Ivashkevich for fruitful discussions and critical reading of the manuscript. We also want to thank D.G. Gaidashev for useful remarks. 


\section{Appendix}

The branching probability of a spanning tree $p_{k}$ is the probability for any site of a random spanning tree to have a coordination number $k$. The effective tool used for evaluating branching probabilities is provided by the Kirchhoff theorem about spanning trees. The method of applying it is not novel. Below we follow the methods outlined in the article [6]. Here we recall its main features. To formulate the Kirchhoff theorem we consider the arbitrarily connected graph $\mathbf{L}$ consisting of $n+1$ sites. Let us fix any site which will be a root. The weight $x_{i j}$ corresponds to each bond of $\mathbf{L}$ edged by the sites $i$ and $j$. Let $\Delta(x)$ be a $n \times n$ matrix:

$$
\Delta_{i j}(x)=\left\{\begin{array}{ccc}
\sum_{k} x_{i k} & , & i=j \\
-x_{i j} & , & i \text { and } j \text { are adjacent sites } \\
0 & , & \text { otherwise }
\end{array}\right.
$$

While the summation is performed over all sites of $G i$ and $j$ run over all sites different from the root. Then, according to the Kirchhoff theorem,

$$
g(x)=\operatorname{det} \Delta(x)
$$

is nothing but a generating function of the rooted spanning trees on this graph. If all $x_{i j}$ are equal to 1 , the matrix

$$
\Delta=\Delta\left(x_{i j}=1\right)
$$

is the discrete Laplacian on graph $\mathbf{L}$ and

$$
N=\operatorname{det} \Delta
$$

gives a total number of the spanning trees on this graph.

For calculating a number of spanning trees on the triangular lattice $L$ whose random site $i$ has $k$ adjacent sites, let us consider the lattices $L^{\prime}$ with the defect in the cell containing site $i$. In other words, we deal with lattices obtained from $L$ by deleting one or several bonds outgoing from $i$. If we leave only one bond connected with $i$, all trees on this new lattice will occupy it. Summing over all possible directions of this bond, we obtain the number of spanning trees the site $i$ of which has only one adjacent site. In the same manner we can link the numbers of spanning trees with other coordination numbers of site $i$ with total numbers of spanning trees on the lattices with a local defect

$$
N_{i}^{\prime}=\sum_{k=1}^{i} \frac{C_{6}^{i} C_{i}^{k}}{C_{6}^{k}} N_{k}
$$

where $N_{k}$ is the number of spanning trees in which the site $i$ has the coordination number $k . N_{k}^{\prime}$ is the sum of total numbers of spanning trees on the lattices obtained from $L$ by deleting all bonds connected with site $i$ except $k$ ones which is taken over all possible positions of these bonds. Dividing $N_{k}$ by the total number of spanning trees, we obtain the branching probabilities. 
Thus, we reduce the problem of searching for branching probabilities search to the calculation of the numbers of spanning trees on the lattices with the defect. To this end, we use the Kirchhoff theorem. According to it, the number of spanning trees on the lattice with a defect is given by the formula

$$
N^{\prime}=\operatorname{det} \Delta^{\prime}
$$

$\Delta^{\prime}$ is the discrete Laplacian on a new lattice. If we refer to the difference between $\Delta$ and $\Delta^{\prime}$ as a defect matrix $\delta$, branching probabilities are expressed through the terms of the following form:

$$
\frac{N^{\prime}}{N}=\frac{\operatorname{det} \Delta^{\prime}}{\operatorname{det} \Delta}=\operatorname{det}(I+G \delta)
$$

$I$ is the unit matrix; $G$ satisfies the matrix equation

$$
\Delta G=I
$$

In the thermodynamic limit $G$ is nothing but the Green function of the Laplace equation on the triangle lattice. For an infinite lattice $G$ depends only on the difference of site coordinates rather than their values

$$
G\left(\mathbf{r}_{\mathbf{1}}-\mathbf{r}_{\mathbf{2}}\right)=\int_{0}^{2 \pi} \int_{0}^{2 \pi} \frac{\cos \left(\alpha\left(x_{1}-x_{2}\right)+\beta\left(y_{1}-y_{2}\right)\right)-1}{1-\frac{1}{3}(\cos \alpha+\cos \beta+\cos (\alpha+\beta))} \frac{d \alpha}{2 \pi} \frac{d \beta}{2 \pi}
$$

Due to defect localization, the matrix $\delta$ has only a finite number of nonzero elements. Thus, we should calculate the $7 \times 7$ matrix determinants. In this way, we obtain the following results:

$$
\begin{gathered}
p_{1}=-\frac{25}{108}+\frac{324 \sqrt{3}}{\pi^{5}}-\frac{540}{\pi^{4}}+\frac{66 \sqrt{3}}{\pi^{3}}+\frac{14}{\pi^{2}}-\frac{55}{12 \sqrt{3} \pi} \\
p_{2}=-\frac{235}{144}-\frac{1620 \sqrt{3}}{\pi^{5}}+\frac{3213}{\pi^{4}}-\frac{702 \sqrt{3}}{\pi^{3}}+\frac{277}{2 \pi^{2}}+\frac{223}{12 \sqrt{3} \pi} \\
p_{3}=\frac{17}{36}+\frac{3240 \sqrt{3}}{\pi^{5}}-\frac{7452}{\pi^{4}}+\frac{2160 \sqrt{3}}{\pi^{3}}-\frac{838}{\pi^{2}}+\frac{241}{2 \sqrt{3} \pi} \\
p_{4}=\frac{2489}{216}-\frac{3240 \sqrt{3}}{\pi^{5}}+\frac{8478}{\pi^{4}}-\frac{2928 \sqrt{3}}{\pi^{3}}+\frac{1489}{\pi^{2}}-\frac{2203}{6 \sqrt{3} \pi} \\
p_{5}=-\frac{115}{9}+\frac{1620 \sqrt{3}}{\pi^{5}}-\frac{4752}{\pi^{4}}+\frac{1854 \sqrt{3}}{\pi^{3}}-\frac{1088}{\pi^{2}}+\frac{3857}{12 \sqrt{3} \pi} \\
p_{6}=\frac{175}{48}-\frac{324 \sqrt{3}}{\pi^{5}}+\frac{1053}{\pi^{4}}-\frac{450 \sqrt{3}}{\pi^{3}}+\frac{569}{2 \pi^{2}}-\frac{355}{4 \sqrt{3} \pi}
\end{gathered}
$$




\section{References}

[1] P. Bak, C. Tang and K. Wiesenfeld, Phys. Rev. Lett. 59 (1987) 381; Phys. Rev. A 38 (1988) 364.

[2] L. Pietronero, A. Vespignani and S. Zapperi, Phys. Rev. Lett. 72 (1994) 1690; A. Vespignani, S. Zapperi and L. Pietronero, Phys. Rev. E 51 (1995) 1711.

[3] V. Loreto, L. Pietronero, A. Vespignani and S. Zapperi, Phys. Rev. Lett. 75 (1995) 465.

[4] E.V. Ivashkevich, Phys. Rev. Lett. 76 (1996) 3368.

[5] D. Dhar, Phys. Rev. Lett. 64 (1990) 1613.

[6] V.B. Priezzhev, J. Stat. Phys. 74 (1994) 955.

[7] V.B. Priezzhev, D.V. Ktitarev and E.V. Ivashkevich, Phys. Rev. Lett. 76 (1996) 2093.

[8] S.N. Majumdar, D. Dhar, J. Phys. A 24 (1991) 475L.

[9] S.N. Majumdar, D. Dhar, Physica A 185 (1992) 129.

[10] S.S. Manna, D. Dhar and S.N. Majumdar, Phys. Rev. A 46 (1992) R4471. 


\section{Figure Captions}

Fig.1: a) Transformation from the lattice $L_{b}$ to $L_{b \sqrt{3}}$. It is performed in such a way that the new lattice is again triangular.

b) Block of three cells of the sublattice $L_{b}$. This block becomes a cell on the new lattice. We show that the directions outgoing from the cells, forming the block, are coupled to the directions of the lattice at the next scale. For example: $x_{1}^{(2)}, x_{2}^{(2)}$ are replaced by $y_{1}$ and $x_{3}^{(2)}, x_{2}^{(3)}$ are replaced by $y_{2}$. The superscript denotes the cell from which the given direction outgoes.

Fig.2: Three types of blocks, whose toppling should be taken into account, and their relaxation schemes. The other blocks can be obtained from these ones by rotations. The letters in the circles denote the height of the cells. $\mathrm{X}$ can take the value A,B,C or D. 


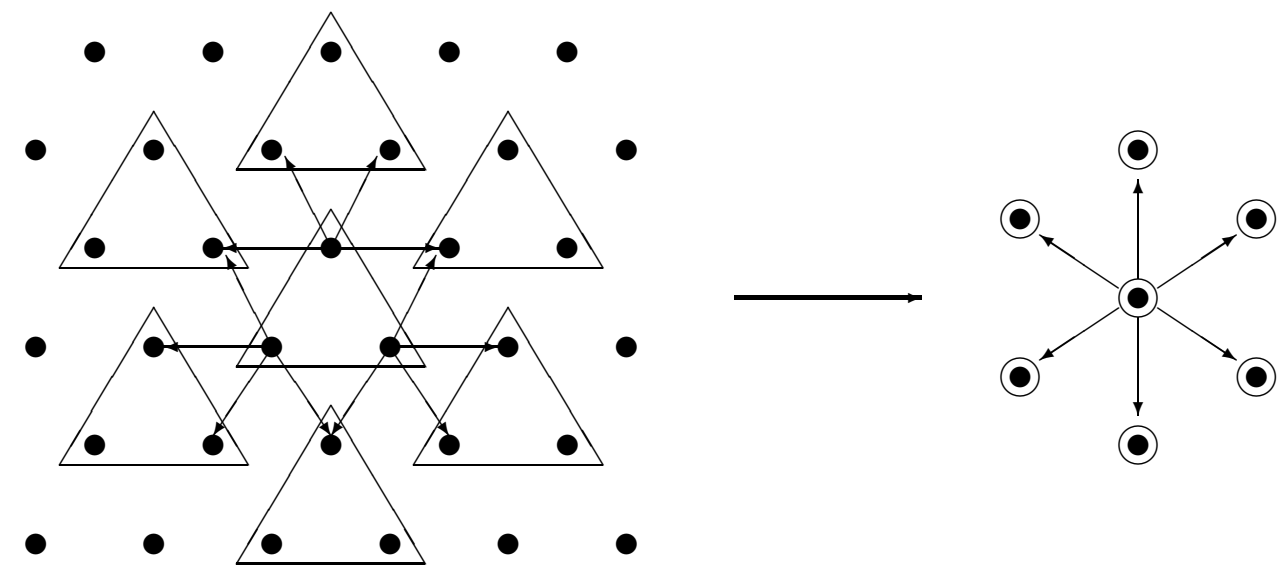

(a)

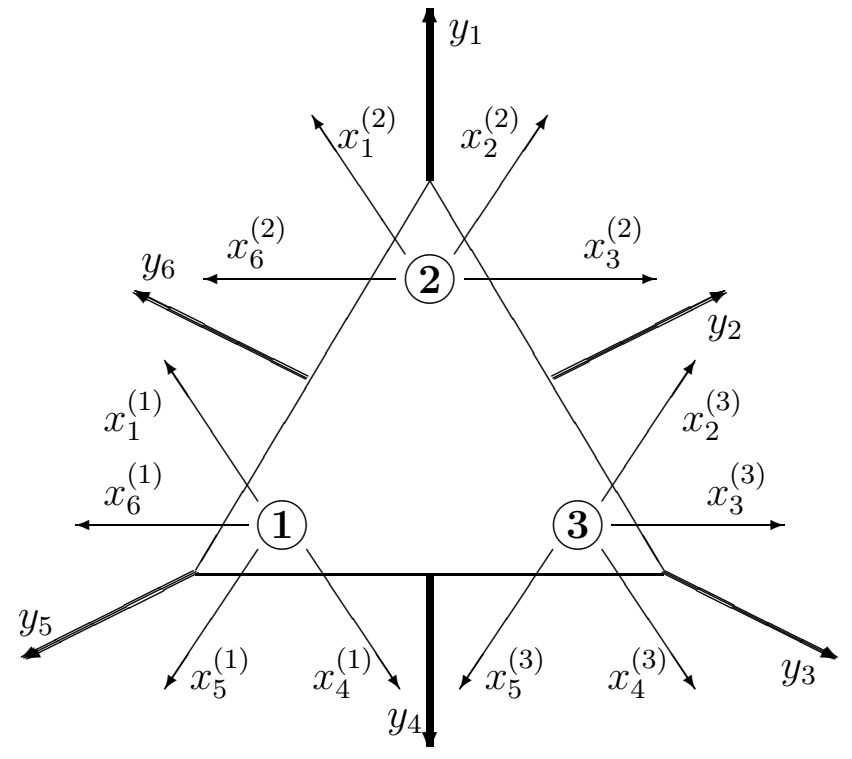

(b)

Fig.1. 
(a)
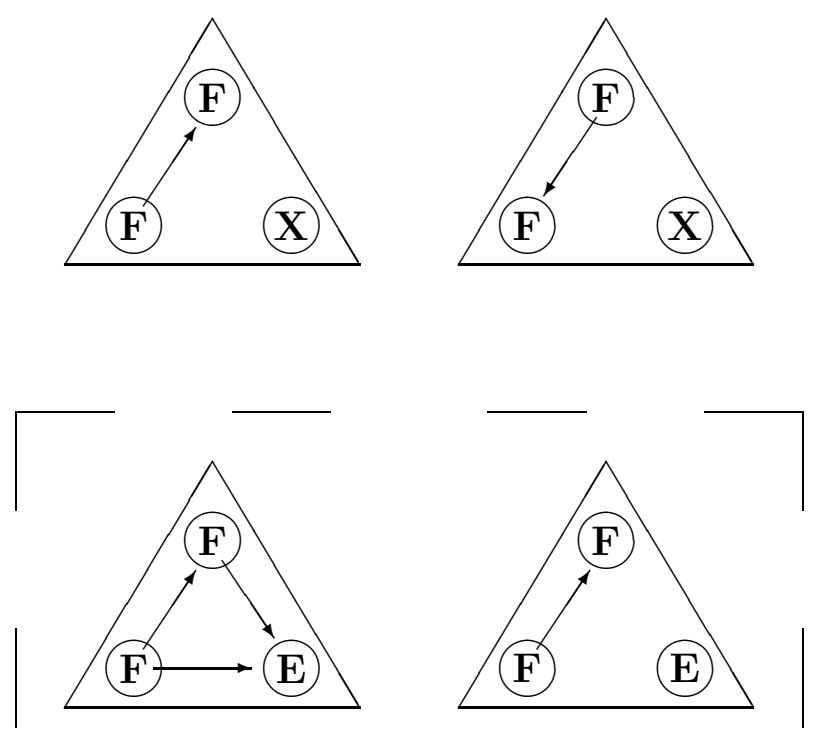

(b)
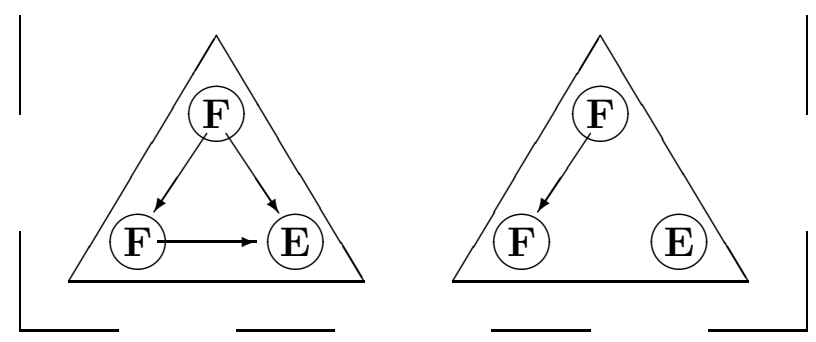

(c)
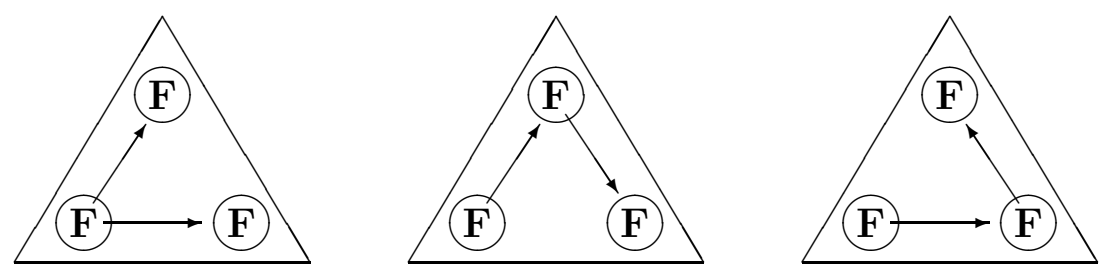

Fig. 2. 